\title{
ISOTOPICALLY CONTROLLED SEMICONDUCTORS
}

\author{
E.E. Haller* \\ Lawrence Berkeley National Laboratory and University of California, Berkeley \\ Berkeley, CA 94720 USA
}

\begin{abstract}
:
Semiconductor bulk crystals and multilayer structures with controlled isotopic composition have attracted much scientific and technical interest in the past few years. Isotopic composition affects a large number of physical properties, including phonon energies and lifetimes, bandgaps, the thermal conductivity and expansion coefficient and spin-related effects. Isotope superlattices are ideal media for self-diffusion studies. In combination with neutron transmutation doping, isotope control offers a novel approach to metal-insulator transition studies. Spintronics, quantum computing and nanoparticle science are emerging fields using isotope control.
\end{abstract}

KEYWORDS: stable isotopes, semiconductors, diffusion, neutron-transmutationdoping, phonons, isotope-superlattices, metal-insulator-transition, spintronics, nanoparticles

\section{Introduction}

The end of the Cold War led to new and unique opportunities for collaborations between laboratories in the countries of the Former Soviet Union (FSU) and the rest of the world. Some laboratories in the FSU countries had accumulated large stocks of highly enriched isotopes of a great number of elements, among them the elements forming semiconductors. Thus started a broad range of investigations involving bulk and thin film multilayer semiconductor structures with highly controlled isotopic composition. In the following sections, a number of the more spectacular results, which were obtained through stable isotope control, will be reviewed and new fields of semiconductor research, which may profit from isotope control, will be discussed briefly.

\section{Phonons and Phonon-Related Properties}

Phonons, the quantized vibrational modes of a crystal lattice, depend very directly on the masses of the isotopes making up the crystal. Using a spring-and-ball analog, we recall that the phonon frequency is proportional to $1 / \sqrt{\mathrm{M}}(\mathrm{M}=$ isotope mass). A direct verification of this dependence has been made by Fuchs et al. ${ }^{(1)}$ in Raman spectroscopy experiments with four of the five stable isotopes of germanium. Approximately $80 \%$ of all the elements have two or more stable isotopes, including the common semiconductors silicon $\left({ }^{28} \mathrm{Si},{ }^{29} \mathrm{Si},{ }^{30} \mathrm{Si}\right)$, germanium $\left({ }^{70} \mathrm{Ge},{ }^{72} \mathrm{Ge},{ }^{73} \mathrm{Ge},{ }^{74} \mathrm{Ge},{ }^{76} \mathrm{Ge}\right)$, gallium arsenide $\left({ }^{69} \mathrm{Ga},{ }^{71} \mathrm{Ga},{ }^{75} \mathrm{As}\right)$ and diamond $\left({ }^{12} \mathrm{C},{ }^{13} \mathrm{C}\right)$. In such multi-isotopic materials, the phonon frequencies depend on an averaged isotopic mass. Formation of extended isotope multilayer structures of germanium (called isotope superlattices) has led to a number of unique results involving zone folding of the phonon dispersion relationship of this well-studied semiconductor ${ }^{(2)(3)}$ (see Fig. 1). In a very

\footnotetext{
* Tel. 510-486-5294

Fax. 510-486-5530

e-mail: EEHALLER@LBL.GOV
} 
recent study, coherent, confined LO phonons generated through femtosecond laser pulses were investigated for the first time in a ${ }^{70} \mathrm{Ge} /{ }^{74} \mathrm{Ge}$ isotope superlattice ${ }^{(4)}$. The isotope superlattices used for these studies are grown by the Molecular Beam Epitaxy (MBE) technique and they typically consist of 2 to 32 alternating atomic layers, each of the two stable isotopes. Raman spectroscopy is used to measure the optical phonon frequencies at the center of the Brillouin zone. Calculations based on the planar force constant mode ${ }^{(5)}$, using the bond polarizability approach ${ }^{(6)}$, accurately reproduce the observed spectra.

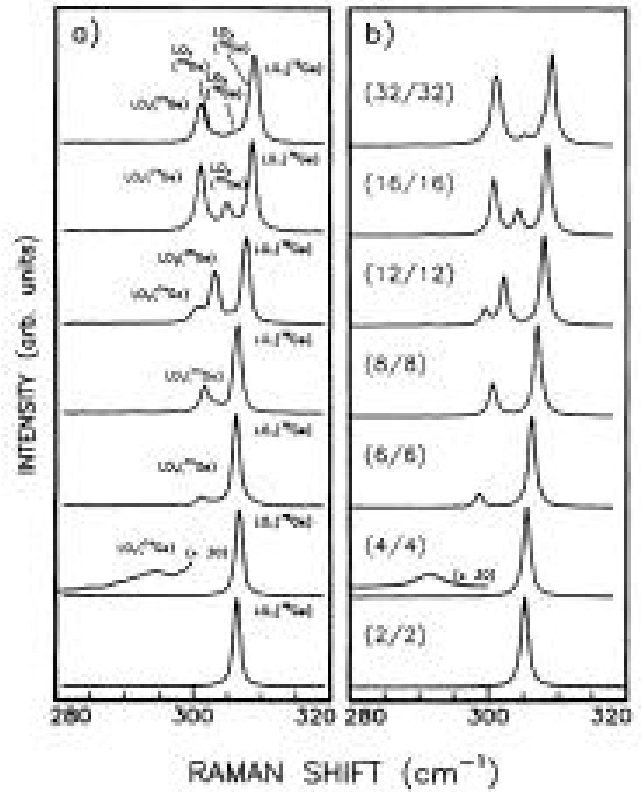

Fig. 1. (a) Measured and (b) calculated Raman spectra for a series of isotopic ${ }^{70}(\mathrm{Ge})_{\mathrm{n}}{ }^{74}(\mathrm{Ge})_{\mathrm{n}}$ SL's showing various confined modes of ${ }^{70} \mathrm{Ge}$ and ${ }^{74} \mathrm{Ge}$ layers. The measurements were performed with the $514.5 \mathrm{~nm}$ line of an $\mathrm{Ar}^{+}$-ion laser at a temperature of $10 \mathrm{~K}$. Calculated Raman peaks have been numerically broadened by $1.2 \mathrm{~cm}^{-1}$ to facilitate comparison with experiment. (after Ref. 2)

Phonons transport heat and are directly responsible for the thermal conductivity. As early as 1942, Pomeranchuk predicted that the thermal conductivity of crystalline solids should depend on isotopic composition ${ }^{(7)}$. Geballe and Hull were first to demonstrate experimentally an increase in the thermal conductivity in isotopically enriched germanium ${ }^{(8)}$. Spectacular thermal conductivity increases have been measured in isotopically enriched diamond ${ }^{(9)}$. Indeed, the low-temperature thermal conductivity of diamond exceeds today's experimentally accessible limits! Isotopically enriched ${ }^{28} \mathrm{Si}$ is currently investigated for its suitability in advanced very high speed integrated circuits (IC). The improved thermal conductivity of enriched ${ }^{28} \mathrm{Si}{ }^{(10)}$ has been shown in model calculations to reduce the highest temperature spots in advanced ICs by several tens of degrees. This more efficient spreading of the dissipated power could be achieved with a 10 to $20 \mu \mathrm{m}$ thick epitaxial layer of ${ }^{28} \mathrm{Si}$ on a silicon wafer of natural isotopic composition.

More subtle, but from a basic science point-of-view important effects related to isotopic composition, are changes in the lattice constant ${ }^{(11)(12)}$, the thermal expansion coefficient ${ }^{(13)}$ and the bandgap ${ }^{(14)}$. Accurate and detailed studies of these physical parameters have been conducted and published for a number of semiconductors.

\section{Doping and Electronic Transport}

The ability to control the electrical conductivity with two types of charge carriers (holes and electrons) is perhaps the most important advantage of semiconductors over other material. While this control is commonly achieved through the introduction of dopant impurities into the semiconductor by a number of different processes (addition of dopants to the melt, ion implantation, diffusion, etc.), there is an unconventional way of doping based on the 
transmutation of semiconductor host isotopes into dopant isotopes. Transmutation can be induced through capture of thermal neutrons. Neutron Transmutation Doping (NTD) is used to dope several hundred tons of ultra-pure silicon with phosphorus according to the following nuclear reaction:

${ }_{14}^{30} \mathrm{Si}(3.10 \%)+\mathrm{n}_{\text {thermal }} \rightarrow{ }_{14}^{31} \mathrm{Si} \frac{\beta}{\mathrm{T} 1 / 2=2.6 \mathrm{hrs}} \rightarrow{ }_{15}^{31} \mathrm{P}+\overline{\mathrm{v}}_{\mathrm{e}} \quad$ (acceptor)

Doping homogeneity and reproducibility resulting from the NTD process are superior to the conventional doping techniques ${ }^{(15)}$. NTD of natural germanium is of special interest because acceptors, donors and double donors are formed after exposure to thermal neutrons.

$$
\begin{aligned}
& { }_{32}^{70} \mathrm{Ge}(20.5 \%)+\mathrm{n}_{\mathrm{th}} \rightarrow{ }_{32}^{71} \mathrm{Ge} \frac{\mathrm{EC}}{\mathrm{T} 1 / 2=11.2 \mathrm{~d}} \rightarrow{ }_{31}^{71} \mathrm{Ga}+\mathrm{v}_{\mathrm{e}} \\
& { }_{32}^{72} \mathrm{Ge}(27.4 \%)+\mathrm{n}_{\mathrm{th}} \rightarrow{ }_{32}^{73} \mathrm{Ge} \\
& { }_{32}^{73} \mathrm{Ge}(7.8 \%)+\mathrm{n}_{\mathrm{th}} \rightarrow{ }_{32}^{74} \mathrm{Ge} \\
& { }_{32}^{74} \mathrm{Ge}(36.5 \%)+\mathrm{n}_{\mathrm{th}} \rightarrow{ }_{32}^{75} \mathrm{Ge} \frac{\beta^{-}}{{ }_{\mathrm{T} 1 / 2}=82.8 \mathrm{~min}} \rightarrow{ }_{33}^{75} \mathrm{As}+\overline{\mathrm{v}}_{\mathrm{e}} \quad \text { (donor) } \\
& { }_{32}^{76} \mathrm{Ge}(7.8 \%)+\mathrm{n}_{\mathrm{th}} \rightarrow{ }_{32}^{77} \mathrm{Ge} \frac{\beta^{-}}{\mathrm{T} 1 / 2=11.3 \mathrm{hrs}} \rightarrow{ }_{33}^{77} \mathrm{As}+\overline{\mathrm{v}}_{\mathrm{e}} \\
& \frac{\beta_{\mathrm{T} 1 / 2}=38.8 \mathrm{hrs}}{{ }_{3}^{-}} \rightarrow{ }_{34}^{77} \mathrm{Se}+\overline{\mathrm{v}}_{\mathrm{e}} \quad \text { (double donor) }
\end{aligned}
$$

Equations 2-6 describe the decay schemes of the five stable Ge isotopes after the capture of a thermal neutron $\left(\mathrm{n}_{\mathrm{th}}\right)$. The natural abundances are given in parentheses after each Ge isotope. EC stands for the electron capture process and $\beta^{-}$for the beta decay. $v_{\mathrm{e}}$ and $\overline{\mathrm{v}}_{\mathrm{e}}$ are the neutrino and the antineutrino respectively.

The transmutation products are germanium acceptors (Ga), donors (As) and double donors (Se). It is evident that changing the relative isotopic composition directly affects the relative dopant concentrations. Growing germanium crystals consisting only of a mixture of ${ }^{70} \mathrm{Ge}$ and ${ }^{74} \mathrm{Ge}$ isotopes and using NTD allows the formation of a continuous series of crystals doped form purely p-type $\left({ }^{70} \mathrm{Ge} 100 \%\right)$ to purely n-type $\left({ }^{74} \mathrm{Ge} 100 \%\right)$ with all the possible compensation ratios between these two extremes. Our group has used NTD with natural Ge to form highly sensitive thermal detectors operating in the Kelvin and milliKelvin temperature range $^{(16)}$ in a large number of far infrared and microwave astronomy and astrophysics experiments ${ }^{(17)-(19)}$. The group of K. Itoh at Keio University has taken advantage of the excellent uniformity and reproducibility of NTD and combined it with the isotope control to study the metal-insulator transition in germanium ${ }^{(20)(21)}$. The quality of these samples have set new standards for this field. Figure 2 shows the precise merging of the relevant parameters $\left[\mathrm{T}_{0}=\right.$ exponential factor in the Shklovskii-Efros hopping conduction theory $\left.{ }^{(22)}\right]$ on the insulating side and the zero-temperature conductivity on the metallic side of a series of samples taken from the same piece of ultra-pure germanium, doped with NTD to different 
acceptor and donor concentrations. The concentration at which the two sides merge is the critical concentration $\mathrm{N}_{\mathrm{C}}=1.856 \times 10^{17} \mathrm{~cm}^{-3}$. The Itoh group has expanded these studies to isotopically controlled germanium samples and to include magnetic fields ${ }^{(23)}$.

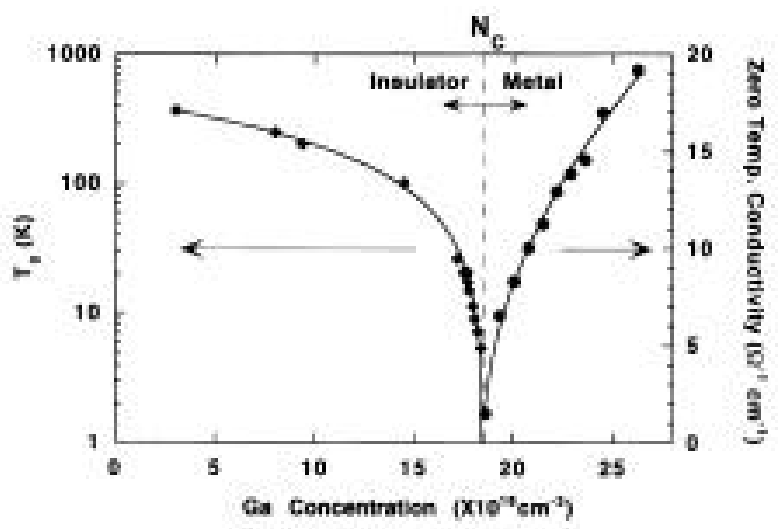

Fig. 2. The left side shows the experimentally determined $\mathrm{T}_{0}$ of 14 insulating samples as a function of $\mathrm{Ga}$ concentration $(\diamond)$. The right side shows the zero-temperature conductivity $\sigma(0)$ (O). Both dependencies meet precisely at the critical concentration $\mathrm{N}_{\mathrm{C}}=1.856 \times 10^{17}$ $\mathrm{cm}^{-3}$. (after Ref. 20)

\section{Diffusion}

The study of diffusion in semiconductors is of vital importance for the formation of $\mathrm{p}$-njunctions, bipolar transistors and Metal-Oxide-Semiconductor (MOS) devices. These are the building blocks of all modern ICs. The shrinking dimensions of ICs and the associated increase in the clock frequencies requires shallower doping depth and higher concentrations, both challenges for diffusion driven processes. The increasing demands on an improved understanding of dopant diffusion processes, together with the availability of isotope multilayer structures of numerous semiconductors, have advanced the field of diffusion science significantly. It is now possible to study the depth dependence of dopant and host lattice isotope concentrations simultaneously using Secondary Ion Mass Spectrometry (SIMS). Typical depth profiles of an AlGaAs/GaAs heterostructure in which the $\mathrm{Ga}$ is isotopically controlled $\left({ }^{27} \mathrm{Al}\right.$ and ${ }^{75} \mathrm{As}$ are both mono-isotopic!) are shown in Fig. $3{ }^{(24)}$ in the as-grown state and after annealing for 30 minutes at $1050^{\circ} \mathrm{C}$.

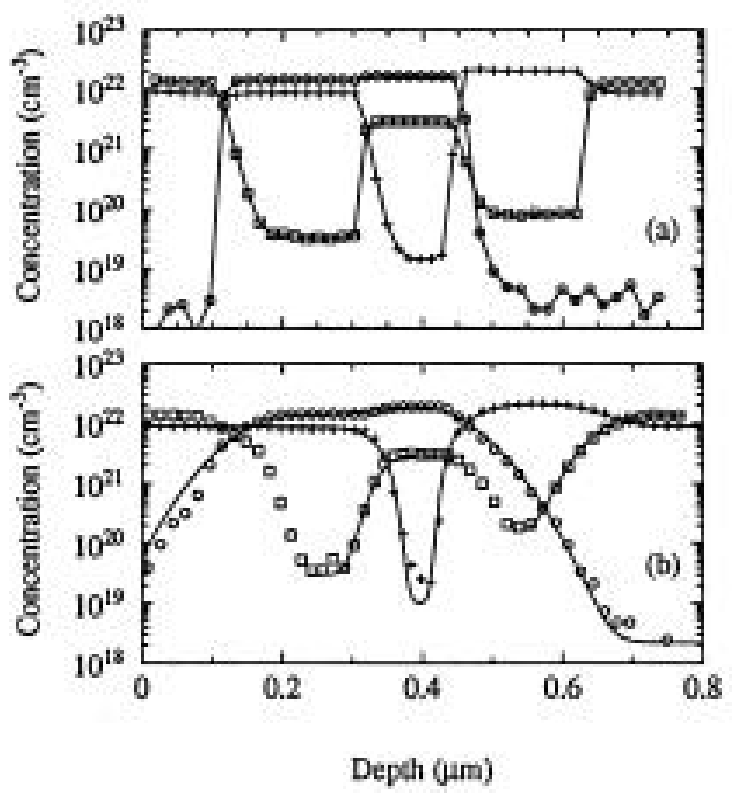

Fig. 3. SIMS depth profiles of $\mathrm{Al}(\mathrm{O}),{ }^{69} \mathrm{Ga}$ $(\square)$, and ${ }^{71} \mathrm{Ga} \quad(+)$ in the as-grown $\mathrm{Al}^{71} \mathrm{GaAs} / \mathrm{Al}^{69} \mathrm{GaAs} /{ }^{71} \mathrm{GaAs}$ heterostructure [see (a)] and after annealing at $1050^{\circ} \mathrm{C}$ for $1800 \mathrm{~s}$ [see (b)]. Solid lines in (a) connect the data to guide the eye. Solid lines in (b) show best fits to the experimental profiles. For clarity, only every fourth data point is plotted in (a) and (b). (after Ref. 24) 
Studies of GaAs self-diffusion have been conducted with donors ( $\mathrm{Si}$ ) and acceptors $(\mathrm{Be})$. The observation that donor doping enhances Ga self-diffusion, while acceptor doping retards it, is direct evidence for a negatively charged native defect, presumably the Ga vacancy, promoting self-diffusion $^{(25)}$. Detailed self-diffusion studies have been conducted with isotope multilayer structures of silicon $^{(26)}$, germanium ${ }^{(27,28)}$, gallium phosphide ${ }^{(29)}$ and most recently with gallium antimonide $^{(30)}$. The last study was conducted with isotope control of both sublattices and yielded the surprising result that $\mathrm{Ga}$ diffuses $\sim 10^{3}$ times faster than $\mathrm{Sb}$ under intrinsic conditions.

\section{Nanoscience and Quantum Computing}

The use of stable isotopes in semiconductor science and technology is by now firmly established and a broad range of very diverse studies have yielded numerous interesting results. Still, it is my belief that we are at the beginning of most interesting uses of isotopically controlled semiconductors. The rapidly expanding field of nanoscience has not made any use of isotope control.

We envision isotopically structured nanoparticles with unique optical or electrical transport properties. Spintronics is using the electron spin for information storage and transmission. Isotope control allows the deliberate introduction or omission of nuclear spins and nuclear magnetic moments (e.g. ${ }^{29} \mathrm{Si}$ ). The nuclear spin carrying isotope can be distributed in the semiconductor matrix at the nanometer level using ion implantation and advanced lithographic techniques. We expect that the full exploitation of isotope control will bring us closer to the realization of quantum computing.

Acknowledgments: This work was supported in part by the Director, Office of Science, Office of Basic Energy Sciences, Division of Materials Sciences, U.S. Department of Energy under Contract No. DE-AC03-76SF00098, by U.S. NSF Grant No. DMR-0109644 and by the Miller Institute for Basic Research in Science. I am indebted to a large number of colleagues who have collaborated extensively with us on isotopically controlled semiconductors.

\section{REFERENCES}

(1) H. D. Fuchs, C. H. Grein, M. Cardona, W. L. Hansen, K. Itoh, and E. E. Haller, Solid State Commun., 82, 225 (1992).

(2) J. Spitzer, T. Ruf, M. Cardona, W. Dondl, R. Schorer, G. Abstreiter, and E. E. Haller, Phys. Rev. Lett., 72, 1565 (1994).

(3) K. Morita, K. M. Itoh, J. Muto. K. Mizoguchi, N. Usami, Y. Shiraki, and E. E. Haller, Proc. Intl. Joint Confr. on Silicon Epitaxy and Heterostructures (IJC-Si), Sept. 13-17, 1999, Miyagi, Japan; Thin Solid Films, 369, 405 (2000).

(4) M. Nakajima, H. Harima, K. Morita, K. M. Itoh, K. Mizoguchi, and E. E. Haller, Phys. Rev. B, 63, R161304 (2001).

(5) B. Jusserand and M. Cardona, in Light Scattering in Solids V, eds. M. Cardona and G. Güntherodt (Springer-Verlag, Berlin, 1989), pp. 49-152.

(6) P. Molinas-Mata and M. Cardona, Phys. Rev. B 43, 9799 (1991).

(7) I. Pomeranchuk, J. Physics (Moscow), 6, 237 (1942).

(8) T.H. Geballe and G. Hull, Phys. Rev., 110, 773 (1958).

(9) L. Wei, P.K. Kuo, R.L. Thomas, T.R. Anthony, and W.F. Banholzer, Phys. Rev. Lett., 70, 3764 (1993). 
(10) T. Ruf, R.W. Henn, M. Asen-Palmer, E. Gmelin, M. Cardona, H.-J. Pohl, G.G. Devyatych, and P.G. Sennikov, Solid State Commun., 115, 243 (2000).

(11) R.C. Buschert, A.E. Merlin, S. Pace, S. Rodriguez, and M.H. Grimsditch, Phys. Rev. B, 38, 5219 (1988).

(12) H. Holloway, K.C. Hass, M.A. Tamor, T.R. Anthony, and W.F. Banholzer, Phys. Rev. $B, 44,7123$ (1991).

(13) V. I. Ozhogin, N. A. Babushkina, L. M. Belova, A. P. Zhernov, E. E. Haller, and K. M. Itoh, J. Exper. Theor. Phys., 88, 135 (1999).

(14) C. Parks, A. K. Ramdas, S. Rodriguez, K. M. Itoh, and E. E. Haller, Phys. Rev. B, 49, 14244 (1994).

(15) See: Proc. Fourth Intl. Conf. on Neutron Transmutation Doping of Semiconductor Materials, National Bureau of Standards, June 1-2, 1982, Gaithersburg, MD. R. D. Larrabee, ed., (Plenum Press 1984).

(16) E. E. Haller, N. P. Palaio, M. Rodder, W. L. Hansen, and E. Kreysa, Proc. Fourth Intl. Conf. on Neutron Transmutation Doping of Semiconductor Materials, National Bureau of Standards, June 1-2, 1982, Gaithersburg, MD. R. D. Larrabee, ed., (Plenum Press 1984), pp. 21-36.

(17) E. Kreysa, H.-P. Gmünd, J. Gromke, C. G. T. Haslam, L. Reichertz, E. E. Haller, J. W. Beeman, V. Hansen, A. Sievers, and R. Zylka, Infrared Phys. Technol., 40, 191 (1999).

(18) A.E. Lange, P.A.R. Ade, J.J. Bock, J.R. Bond, J. Borrill, A. Boscaleri, K. Coble, B.P. Crill, P. de Bernardis, P. Farese, P. Ferreira, K. Ganga, M. Giacometti, E. Hivon, V.V. Hristov, A. Iacoangeli, A.H. Jaffe, L. Martinis, S. Masi, P.D. Mauskopf, A. Melchiorri, T. Montroy, C.B. Netterfield, E. Pascale, F. Piacentini, D. Pogosyan, S. Prunet, S. Rao, G. Romeo, J.E. Ruhl, F. Scaramuzzi, and D. Sforna, Phys. Rev. D, 63, 42001 (2001).

(19) W. S. Holland, P. A. R. Ade, M. J. Griffin, I. D. Hepburn, D. G. Vickers, C. R. Cunningham, P. R. Hastings, W. K. Gear, W. D. Duncan, T. E. C. Baillie, E. E. Haller, and J. W. Beeman, Intl. J. Infrared and MM Waves, 17, 669 (1996).

(20) K. M. Itoh, E. E. Haller, J. W. Beeman, W. L. Hansen, J. Emes, L. A. Reichertz, E. Kreysa, T. Shutt, A. Cummings, W. Stockwell, B. Sadoulet, J. Muto, J. W. Farmer, and V. I. Ozhogin, Phys. Rev. Lett., 77, 4058 (1996).

(21) M. Watanabe, Y. Ootuka, K.M. Itoh, and E.E. Haller, Phys. Rev. B, 58, 9851 (1998).

(22) B.I. Shklovskii and A.L. Efros, in Electronic Properties of Doped Semiconductors, Solid State Series, Vol. 45 (Springer-Verlag, Berlin, 1984).

(23) M. Watanabe, K.M. Itoh, Y. Ootuka, and E.E. Haller, Phys. Rev. B, 60, 15817 (1999).

(24) H. Bracht, E. E. Haller, K. Eberl, and M. Cardona, Appl. Phys. Lett., 74, 49 (1999).

(25) H. Bracht, M. Norseng, E. E. Haller, K. Eberl, and M. Cardona, Solid State Commun., 112, 301 (1999).

(26) H. Bracht, E. E. Haller, and R. Clark-Phelps, Phys. Rev. Lett., 81, 393 (1998).

(27) H. Fuchs, W. Walukiewicz, E. E. Haller, W. Dondl, R. Schorer, G. Abstreiter, A. I. Rudnev, A. V. Tikhomirov, and V. I. Ozhogin, Phys. Rev. B, 51, 16817 (1995).

(28) E. Silveira, W. Dondl, G. Abstreiter, and E. E. Haller, Proc. Eighth International Conference on Modulated Semiconductor Structures MSS8, Santa Barbara, CA, USA, 14-18 July 1997), Physica E, 2, 291 (1998).

(29) Lei Wang, J. A. Wolk, L. Hsu, E. E. Haller, J. W. Erickson, M. Cardona, T. Ruf, J. P. Silveira, and F. Briones, Appl. Phys. Lett., 70, 1831 (1997).

(30) H. Bracht, S. P. Nicols, W. Walukiewicz, J. P. Silvera, F. Briones, and E. E. Haller, Nature, 408, 69 (2000). 